\title{
Walking-based activity and sedentary behavior in Saudi males with chronic obstructive pulmonary disease
}

\author{
Mohammed M. Alyami, PhD, Sue C. Jenkins, PhD, Kylie Hill, PhD.
}

\begin{abstract}

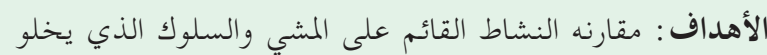

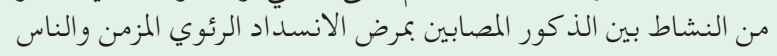

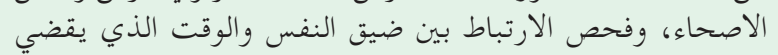

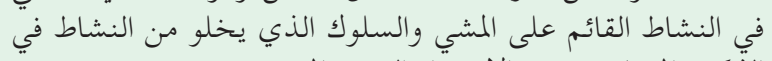

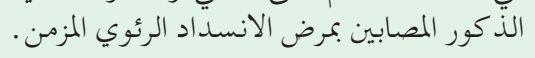

الطريقة : هذه در اسه مقطعيه شملت 30 ذ ذكر مصابين بمرض الانسدار

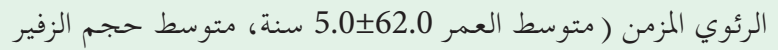

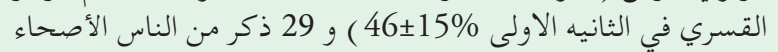

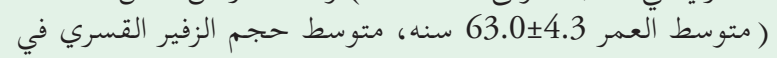

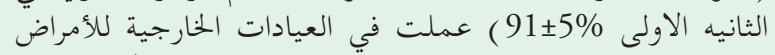

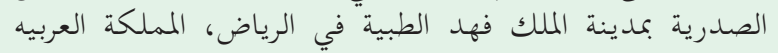

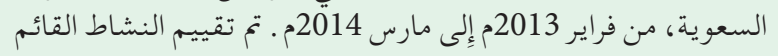

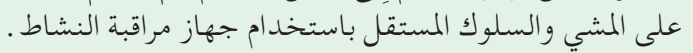

النتائج: الذكور المصابين بمرض الانسداد الرئوي المزمن يقضون وقت المباد

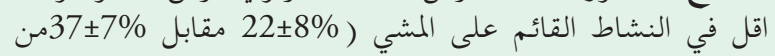
ساعات الإستيقاظ ؛

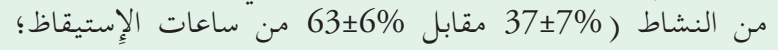
بين 0.001

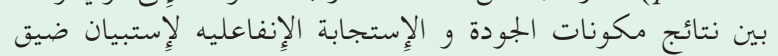

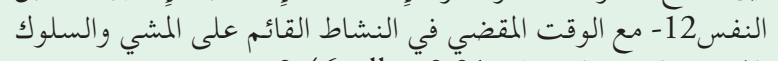

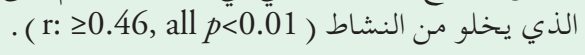

الحخاتمه : أظهرت الدراسة أن الذكور المصابين بمرض الإنسداد الرئوي

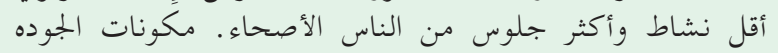

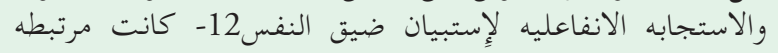

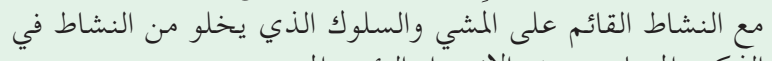

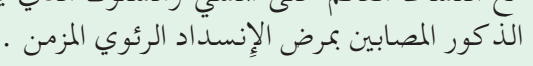

Objectives: To compare walking-based activity and sedentary behavior between males with chronic obstructive pulmonary disease (COPD) and healthy controls and to examine the association between dyspnea with time spent in walking-based activity and sedentary behavior in males with COPD.
Methods: This cross-sectional study of 30 males with COPD (age 62.0 \pm 5.0 years; forced expiratory volume in one second [FEV1] $46 \pm 15 \%$ predicted) and 29 healthy controls (age 63.0 \pm 4.3 years; FEV1 91 $\pm 5 \%$ predicted) was conducted at the outpatient pulmonary clinics at King Fahad Medical City, Riyadh, Kingdom of Saudi Arabia between February 2013 and March 2014. Walking-based activity and sedentary behavior were evaluated using an activity monitor.

Results: Participants with COPD spent less time engaged in walking-based activity $(22 \pm 8 \%$ versus $37 \pm 7 \%$ of waking hours; $p<0.001)$ and more time engaged in sedentary behavior $(78 \pm 8 \%$ versus $63 \pm 6 \%$ of waking hours; $p<0.001)$ than healthy controls. In males with COPD, moderate to strong associations were found between the scores of the quality and emotional response components of the Dyspnea-12 (D-12) questionnaire and time spent engaged in walking-based activity and sedentary behavior $(\mathrm{r}: \geq 0.46$, all $p<0.01)$.

Conclusion: Males with COPD were less active and more sedentary than healthy controls. The D-12 components were associated with walking-based activity and sedentary behavior in males with COPD.

Saudi Med J 2018; Vol. 39 (5): 506-513

doi: 10.15537/smj.2018.5.22043

From the The School of Physiotherapy and Exercise Science (Alyami, Jenkins, Hill), Curtin University; from the Department of Physiotherapy (Jenkins), Sir Charles Gairdner Hospital; from the Institute for Respiratory Health (Jenkins, Hill), Perth, Australia; and from the Faculty of Medicine (Alyami), Batterjee Medical College, Jeddah, Kingdom of Saudi Arabia.

Received 16th January 2018. Accepted 27th March 2018.

Address correspondence and reprint request to: Dr. Mohammed M. Alyami, Batterjee Medical College, Jeddah, Kingdom of Saudi Arabia. E-mail:alyami.moh@hotmail.com

ORCID ID: orcid.org/0000-0001-8658-7305 
I $\mathrm{n}$ health and sickness, numerous health benefits are associated with regular participation in physical activity of moderate to vigorous intensity. ${ }^{1}$ There are robust data demonstrating that people with chronic obstructive pulmonary disease (COPD) engage in less physical activity than healthy controls. ${ }^{2,3}$ In people with COPD, less participation in physical activity has been associated with several factors, including more severe dyspnea upon exertion, airflow obstruction and static lung hyperinflation, less autonomous motivation for exercise, lower exercise capacity, greater impairment of peripheral muscle force, use of long-term oxygen therapy, a higher number of an acute exacerbation of COPD (AECOPD) in the previous year, and greater feelings of depression. ${ }^{4}$ Although several methods can quantify the intensity of physical activity undertaken during daily life, cadence (steps/minute) is a walking parameter that is considered a logical surrogate for intensity. ${ }^{5}$ Several laboratory-based studies have reported that intensity increases with cadence, so $\geq 100$ steps/ minute seem to be a threshold equivalent to physical activity of moderate-to-vigorous intensity (equivalent to $\geq 3$ metabolic equivalent units) in adults. ${ }^{5-8}$ In adults and people with COPD, increased time spent engaged in sedentary behavior is associated with higher mortality risk. ${ }^{9}{ }^{10}$ Compared with healthy controls, people with COPD spend 1.4 times more waking hours in sitting and reclining positions. ${ }^{3,4,11}$ Prolonged sedentary behavior is associated with factors such as lower exercise capacity, less autonomous motivation for exercise, use of long-term oxygen therapy, and a higher rate of exacerbation in the past year in people with COPD. ${ }^{4}$ To date, studies that have assessed the level of physical activity in the Saudi population have used subjective methods (for example, questionnaires). ${ }^{12-15}$ However, quantifying physical activity (or sedentary behavior) using subjective measures has been shown to be inaccurate, particularly in older people, when compared with objective measures (for example, pedometers or accelerometers). ${ }^{16}$ No information on physical activity and sedentary behavior in Saudi nationals with COPD obtained using robust objective methods has been reported. Therefore, the aim of this study was to compare walking-based activity and sedentary behavior between males with COPD and healthy controls. The

Disclosure. Authors have no conflict of interests, and the work was not supported or funded by any drug company. study also examined the association between dyspnea and time spent engaged in walking-based activity and sedentary behavior in males with COPD.

Methods. This study employed a cross-sectional design. Data collection was undertaken between February 2013 and March 2014. Approval to conduct the study was granted by the Institutional Review Board of King Fahad Medical City, Saudi Arabia (approval number 12/312) and the Human Research Ethics Committee of Curtin University, Australia (approval number HR 24/2013).

Cultural issues made it difficult to recruit females to this study, and therefore only males were targeted. Males with COPD were recruited from the outpatient pulmonary clinics at King Fahad Medical City in Riyadh, Kingdom of Saudi Arabia if they: 1) were aged $\geq 40$ years; 2 ) had a diagnosis of COPD defined as post-bronchodilator forced expiratory volume in one second $\left(\mathrm{FEV}_{1}\right) /$ forced vital capacity $(\mathrm{FVC})<0.7$ and predicted post-bronchodilator $\mathrm{FEV}_{1}<80 \% ;{ }^{17} 3$ ) were clinically stable (defined as no change in medication use over the previous 2 months); and 4 ) did not require supplemental oxygen. Further, only those who were retired (for example, no longer had paid employment) were included. Consistent with earlier work, this criteria was used in order to minimize any possible confounding influence that engaging in paid work may have had on their participation in daily physical activity and sedentary behavior. ${ }^{3}$

For the purpose of comparison, healthy males were recruited through distribution of flyers at mosques and discussion with the family members of COPD patients. The inclusion criteria for this group were as follows: 1) absence of a serious health condition; 2) spirometry within normal limits; and 3) retired. The exclusion criteria for both groups were as follows: 1) presence of any comorbid condition thought to adversely affect one's capacity to participate in physical activity, such as uncontrolled cardiovascular disease or severe musculoskeletal condition; 2) use of a gait aid; and 3) inability to read and write the Arabic language.

In order to increase the likelihood that the COPD and healthy controls were balanced in terms of age, males with COPD were recruited first and the mean age and standard deviation (SD) of the sample were determined. Thereafter, healthy people were recruited if they were aged within \pm 1 SD of the mean age of the COPD group.

Protocol. Participants with COPD were required to attend one 2-hour session. During this session, airflow obstruction, dyspnea, and 6-minute walk 
distance (6MWD) were measured. Dyspnea was measured using the Dyspnea-12 questionnaire (D-12). Participants' age, height, and weight were recorded. Upon completion of this session, each participant was fitted with a StepWatch Activity Monitor (SAM; Cyma Corporation, Manchester, Connecticut, USA) and was instructed to wear the device during waking hours for 8 consecutive days, except when showering or swimming. Participants were asked to maintain their usual daily physical activity while wearing the SAM.

The protocol for the healthy control group was similar to that for the COPD group. However, participants in this group were not required to complete the D-12 or perform the 6-minute walk test (6MWT).

Measurements. Walking-based activity and sedentary behavior. Walking-based activity and sedentary behavior were measured using the SAM, which is a small monitor that attaches to the right ankle with a Velcro strap and responds to time, acceleration, and position. The SAM has been validated as an accurate step counter for people with COPD, irrespective of walking speed or use of a wheeled walker. ${ }^{18}$ The height of each participant was entered into the SAM software, and the settings related to "range of walking speed" was selected as "moderate" and the settings related to "leg motion" was selected as "normal".

Dyspnea. Dyspnea was measured using the Arabic D-12, which comprises 12 items that assess the severity of dyspnea. ${ }^{19}$ Each item is graded in terms of its severity using a 4-point scale ranging from none (score $=0)$ to severe (score $=3)$, with higher scores indicating more severe dyspnea. ${ }^{19}$ Of the 12 items, 7 relate to the quality of dyspnea and 5 relate to the emotional response to dyspnea.

Lung function. Post-bronchodilator $\mathrm{FEV}_{1}$ and FVC were measured using an Easy One Spirometer (NDD Medical Technologies, Massachusetts, USA) in accordance with the guidelines of the American Thoracic Society. ${ }^{20}$ Data were expressed in absolute values and as a percentage of the predicted values that were previously established in a local population. ${ }^{21}$

Functional exercise capacity. Functional exercise capacity for participants with COPD was evaluated using the 6MWD according to a protocol based on recommendations made by the European Respiratory and American Thoracic Societies. ${ }^{22}$ The 6MWD was performed over a 45-meter level straight course within an enclosed corridor. Data from this test were expressed in absolute values and as a percentage of the predicted values previously established in an international sample. ${ }^{23}$
Sample size. A previous European study that measured walking time in people with COPD and healthy controls reported that those with COPD participated in walking for $44 \pm 26$ (mean \pm SD) minutes/ day and healthy controls participated in walking for $81 \pm 26$ minutes/day. ${ }^{3}$ These data suggest that people with COPD were, on average, $46 \%$ less active than their retired, healthy age- and gender-matched counterparts. ${ }^{3}$ For the current study, a more modest difference (for instance, a $25 \%$ reduction in physical activity) was expected between Saudi nationals with COPD and healthy controls, because it was expected that the general population of Saudis would be less active than those living in Western countries. ${ }^{15}$ The weather in Saudi Arabia tends to be very hot in the summer and cold in the winter, which limits opportunities for outdoor physical activity. Data from an earlier study that measured the level of physical activity in Saudi nationals using the International Physical Activity Questionnaire revealed that they generally participate in little physical activity. ${ }^{12}$ Because a European study demonstrated that healthy people walked for $81 \pm 26$ minutes each day, a $25 \%$ reduction is equivalent to 20 minutes ( $25 \%$ of 81 minutes $=20$ minutes). Assuming the same SD reported in the European study, in order to detect a difference in walking time of $20 \pm 26 \mathrm{~min} /$ day between the COPD and healthy control groups $(\alpha=0.05,1-\beta=0.8)$, a sample size of 28 was needed for each group.

Stepwatch data management. Data recorded by the SAM were exported to a database and the number of steps for each minute was doubled because the SAM records only steps taken by the right leg. The outcomes of monitoring physical activity in people with moderate to very severe COPD did not differ between weekdays and weekends. ${ }^{24}$ Therefore, to be included in these analyses, participants needed to contribute data over a minimum of 5 days, regardless of whether those days fell on the weekday or weekend, with a minimum wear time of 10 hours per day. ${ }^{25,26}$ For each day, wear time was calculated as the difference in time between the first and last minute during which steps were recorded.

Walking-based activity was defined as $\geq 2$ steps $/ \mathrm{min}$ and sedentary behavior was defined as 0 steps $/ \mathrm{min} .{ }^{27,28}$ For each participant, each minute of data for each day was categorized into the following bands: 1) 2-18 steps/min (incidental movement); 2) 20-38 steps/min (sporadic movement); 3) 40-58 steps/min (purposeful steps); 4) 60-78 steps/min (slow walking); 5) 80-98 steps/min (medium walking); 6) 100-118 steps/min (brisk walking); and 7) $\geq 120$ steps/min (fast walking). Similar cadence bands, albeit arbitrary, were used by the 
National Health and Nutrition Examination Survey (NHANES) to explore differences in walking-based activity in a general population. ${ }^{27}$ For each day, time spent engaged in walking-based activity and sedentary behavior as well as the time spent in each cadence band was expressed both in minutes and as a percentage of total wear time. The latter was normalized for differences in wear time between participants. Then, data were averaged between days.

Statistical analyses. Statistical analyses were performed using the Statistical Package for Social Sciences (SPSS version 21.0, IBM Corp., Armonk, NY, USA). The distribution of data was examined using the Shapiro-Wilk test. Data that did not follow a normal distribution were analyzed using nonparametric statistical tests. Thereafter, data were compared between the 2 groups using either independent-sample t-tests for parametric data or Mann-Whitney U tests for nonparametric data. The chi-squared statistic was used to compare the proportion of participants who accumulated $\geq 7,000$ steps/day, the minimum recommended steps per day. ${ }^{1}$

Associations between the components of the D-12 and time spent engaged in walking-based activity and sedentary behavior in participants with COPD were examined using Pearson's correlations for parametric or Spearman's correlations for nonparametric data. The coefficient of determination for the association between emotional response to dyspnea and time spent engaged in walking-based activity and sedentary behavior was calculated. For all analyses, $p<0.05$ was considered significant. Data are expressed as mean \pm SD unless otherwise stated.

Results. Thirty-four participants with COPD were approached to participate in this study. Their characteristics are presented in Table 1. Data from 4 of these participants (12\%) were not included in the analyses because the participants were hospitalized with AECOPD during the monitoring period $(n=1)$, the accumulated wear time was insufficient $(\leq 10$ hours/day; $\mathrm{n}=1)$, or the participant did not return the SAM $(\mathrm{n}=2)$.

Thirty-two healthy males were approached to participate in this study. Their characteristics are presented in Table 1. Data from three of these participants (9\%) were not included in the analyses because they did not return the SAM.

Table 2 presents the average total time participants with COPD and healthy controls spent engaged in walking-based activity ( $\geq 2$ steps/min) and sedentary behavior ( 0 steps $/ \mathrm{min}$ ), expressed as minutes. Compared with healthy controls, participants with COPD: 1) spent fewer waking hours engaged in walking-based activity $(37 \pm 7 \%$ versus $22 \pm 8 \%$ of waking hours; $p<0.001$; Figure 1), a difference that was equivalent to $15 \pm 12 \%$ of waking hours or $131 \pm 105$ minutes; 2) spent more waking hours engaged in sedentary behavior $(63 \pm 6 \%$ versus $78 \pm$ $8 \%$ of waking hours; $p<0.001$; Figure 1), a difference that was equivalent to $15 \pm 13 \%$ of waking hours or $109 \pm 103$ minutes; 3$)$ accumulated fewer daily steps $(7,472 \pm 2,258$ versus $4,124 \pm 2,039$ steps/day; $p<0.001)$; and 4 ) spent fewer minutes (Table 2) and a lower percentage of waking hours (Figure 2) engaged in walking-based activity at all cadence bands $(<100$ steps/min; $p<0.05)$. Additionally, fewer participants achieved the minimum recommended steps per day, 7,000 (34 \% versus 7\%; $p<0.001) .{ }^{1}$

Moderate associations were found between the score for the quality component of the D-12 and time (expressed both as minutes and percentage of waking hours) spent engaged in walking-based activity (all

Table 1 - Characteristics of 34 participants with chronic obstructive pulmonary disease.

\begin{tabular}{|c|c|c|}
\hline Variables & $\begin{array}{c}\text { COPD participants } \\
(\mathrm{N}=34) \\
\text { mean } \pm \mathrm{SD}\end{array}$ & $\begin{array}{c}\text { Healthy controls } \\
(\mathrm{N}=32) \\
\text { mean } \pm \mathrm{SD}\end{array}$ \\
\hline Age, years & $61.9 \pm 5.2$ & $63.1 \pm 3.6$ \\
\hline BMI, $\mathrm{kg} / \mathrm{m}^{-2}$ & $27.9 \pm 4.4$ & $28.4 \pm 3.8$ \\
\hline Smoking, pack/years & $48.3 \pm 18.3$ & $0.9 \pm 3.1$ \\
\hline $\mathrm{FEV}_{1}, \mathrm{~L}$ & $1.41 \pm 0.55$ & $2.60 \pm 0.31$ \\
\hline $\mathrm{FEV}_{1}, \%$ predicted & $46 \pm 16.0$ & $91 \pm 5$ \\
\hline FVC, L & $2.51 \pm 0.64$ & $3.09 \pm 0.36$ \\
\hline FVC, $\%$ predicted & $67 \pm 14$ & $88 \pm 6$ \\
\hline $\mathrm{FEV}_{1} / \mathrm{FVC}, \%$ & $55 \pm 12$ & $84 \pm 6$ \\
\hline D-12 total score & $19.1 \pm 3.5$ & \\
\hline Quality component score & $10.9 \pm 2.4$ & \\
\hline Emotional response component score & $8.2 \pm 1.6$ & \\
\hline 6MWD, m & $347 \pm 75$ & \\
\hline 6MWD, \% predicted & $63 \pm 12$ & \\
\hline \multicolumn{3}{|l|}{ Smoking status (\%) } \\
\hline Current smoker & $18(53)$ & $4(13)$ \\
\hline Ex-smoker & $16(47)$ & $2 \quad(6)$ \\
\hline Never smoked & $0 \quad(0)$ & $26(81)$ \\
\hline \multicolumn{3}{|l|}{ Comorbidities (\%) } \\
\hline Hypertension & $20 \quad(59)$ & $19 \quad(59)$ \\
\hline Diabetes mellitus & $16(47)$ & $13(41)$ \\
\hline Stable heart disease & $7(21)$ & $4(13)$ \\
\hline GORD & $23(68)$ & $16(50)$ \\
\hline Dyslipidemia & $17(50)$ & $10(31)$ \\
\hline Others & $18 \quad(53)$ & $7(22)$ \\
\hline \multicolumn{3}{|l|}{ GOLD grades (\%) } \\
\hline 2 (moderate) & $13(38)$ & - \\
\hline 3 (severe) & $11 \quad(32)$ & - \\
\hline 4 (very severe) & $10 \quad(30)$ & - \\
\hline \multicolumn{3}{|c|}{$\begin{array}{l}\text { SD - standard deviation, BMI - body mass index, D-12 - dyspnea-12, } \\
\text { FEV } 1 \text { - forced expiratory volume in one second, FVC - forced vital } \\
\text { capacity, GOLD - global initiative for chronic obstructive lung disease, } \\
\text { GORD - gastro-esophageal reflux disease, 6MWD - 6-minute walk } \\
\text { distance. *Significant difference between groups. }\end{array}$} \\
\hline
\end{tabular}


Table 2 - Comparison of walking-based activity and sedentary behavior between groups, expressed in minutes.

\begin{tabular}{lcccc}
\hline Activity & Steps/min & $\begin{array}{c}\text { COPD participants }(\mathbf{n}=30) \\
\text { mean } \pm \text { SD (min) }\end{array}$ & $\begin{array}{c}\text { Healthy controls }(\mathbf{n}=29) \\
\text { mean } \pm \text { SD (min) }\end{array}$ & $P$-value \\
\hline Walking-based activity & $\geq 2$ & $170 \pm 58$ & $302 \pm 53$ & $<0.001^{*}$ \\
Sedentary behavior & 0 & $629 \pm 80$ & $520 \pm 60$ & $<0.001^{*}$ \\
Walking-based activity in cadence bands & & & $<0.001^{*}$ \\
Incidental movement & 2 to 18 & $104 \pm 29$ & $164 \pm 33$ & $<0.001^{*}$ \\
Sporadic movement & 20 to 38 & $46 \pm 20$ & $87 \pm 21$ & $<0.001^{*}$ \\
Purposeful steps & 40 to 58 & $14 \pm 10$ & $34 \pm 9$ & $<0.001^{*}$ \\
Slow walking & 60 to 78 & $5 \pm 4$ & $12 \pm 4$ & $0.008^{*}$ \\
Medium walking & 80 to 99 & $1 \pm 2$ & $4 \pm 3$ & 0.163 \\
Brisk walking & 100 to 118 & $0 \pm 1$ & $1 \pm 1$ & 0.400 \\
Fast locomotion & $\geq 120$ & $0 \pm 0$ & $0 \pm 1$ & \\
\hline
\end{tabular}

Data are presented as mean \pm standard deviation

min - minutes, SD - standard deviation, COPD - chronic obstructive pulmonary disease.

*Significant difference between groups

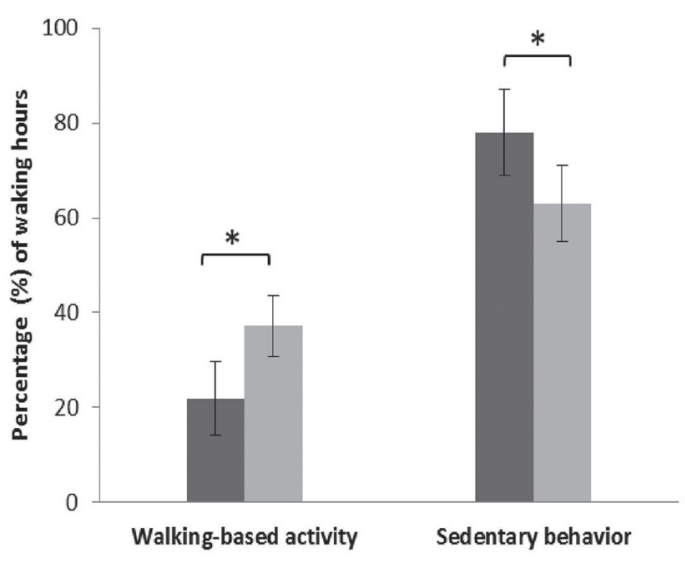

Figure 1 - Comparison of walking-based activity and sedentary behavior between participants with chronic obstructive pulmonary disease (COPD) and healthy controls, expressed as a percentage of waking hours. Data are expressed as mean \pm standard deviation. *Statistically significant difference $(p<0.05)$.

$\mathrm{r}: \geq-0.52, p<0.01$ ) and sedentary behavior (all $\mathrm{r}: \geq 0.46$, $p<0.01$ ). Strong associations were observed between the score of the emotional response component of the D-12 and time (expressed both as minutes and percentage of waking hours) spent engaged in walkingbased activity (all $\mathrm{r}$ : $\geq-0.63, p<0.01$ ) and sedentary behavior (all $\mathrm{r}: \geq 0.66, p<0.01$ ). The total scores of the D-12 were strongly associated with time (expressed both as minutes and percentage of waking hours) spent engaged in walking-based activity (all $\mathrm{r}: \geq-0.72$, $p<0.01$ ) and sedentary behavior (all $\mathrm{r}: \geq 0.66, p<0.01$ ). The coefficient of determination for the association between emotional response to dyspnea and time

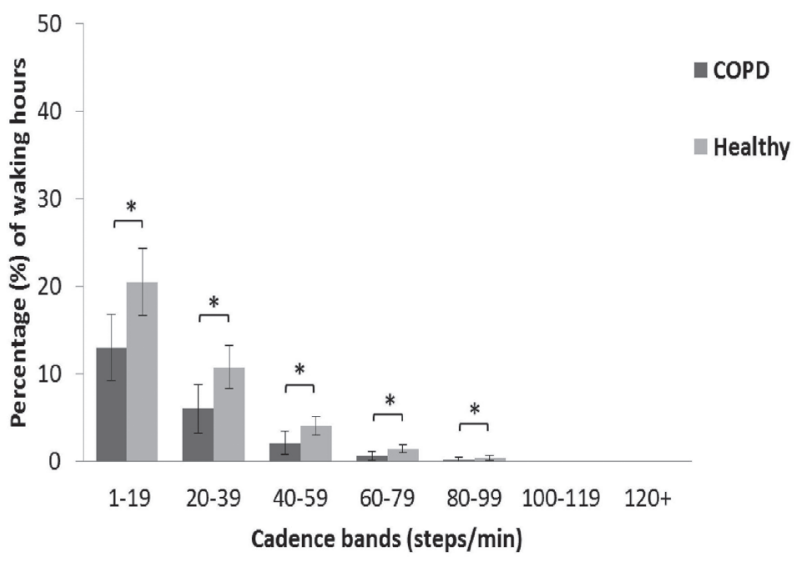

Figure 2 - Comparison of walking-based activity, divided into cadence bands, between participants with chronic obstructive pulmonary disease (COPD) and healthy controls, expressed as a percentage of waking hours. Data are expressed as mean \pm standard deviation. ${ }^{*}$ Statistically significant difference $(p<0.05)$.

spent engaged in walking-based activity was 0.40 and sedentary behavior was 0.44 .

Discussion. This study demonstrated that males with COPD spent fewer waking hours engaged in walking-based activity and more time engaged in sedentary behavior compared to healthy controls. Further, moderate to strong associations were found between the components of the D-12 and time spent engaged in walking-based activity and sedentary behavior in males with COPD.

This is the first study to report data concerning the walking-based activity and sedentary behavior of 
males with COPD and compare it with data obtained from healthy controls in Saudi Arabia. This study demonstrated that, compared to healthy controls, males with COPD spent $15 \pm 12 \%$ fewer waking hours engaged in walking-based activity and $15 \pm 13 \%$ more waking hours engaged in sedentary behavior. Similar findings were reported for people with COPD living in Belgium and Brazil. ${ }^{3,29}$ The average daily step count of males with COPD in this study $(4,124 \pm 2,039$ steps/ day) was noticeably less than the minimum of 7,000 steps generally recommended for older adults. ${ }^{1}$ The amount of daily steps accumulated by males with COPD in this study was similar to that reported for people with COPD from Canada (4,378 $\pm 2,105$ steps/day)and the Netherlands $\left(4,472 \pm 2,716\right.$ steps/day). ${ }^{30,31}$ Compared to healthy controls, the proportion of males with COPD (7\%) who accumulated an average of $\geq 7,000$ steps per day was lower than that of healthy controls (34\%). These findings demonstrate the importance of designing interventions that aim to increase the number of daily steps in males with COPD in order to meet the recommended amount of physical activity necessary to promote and maintain health.

In this study, males with COPD spent a higher proportion $(78 \%)$ of their waking hours engaged in sedentary behavior than people with COPD from Belgium (64\%) and Brazil (56\%). 3,29 The discrepancy in the proportion of sedentary time between this study and previous studies may reflect ethnic, geographical, environmental, cultural, and lifestyle factors unique to Saudi Arabia that impact participation in physical activity. It is important to note that the discrepancy may also have been due to the use of different activity monitors and different definitions of sedentary behavior. $^{3,29}$ The present study used the SAM and defined sedentary behavior as a pragmatic cutoff of 0 steps/min. This may have incorrectly categorized time standing still as sedentary behavior. In contrast, in studies of people with COPD from Belgium and Brazil, the Dynaport accelerometer (McRoberts BV, The Hague, Netherlands) was used to quantify the amount of time spent engaged in sedentary behavior, which was defined as the time spent in a sitting or reclining position. $3,25,29$

This is the first study to report data on the time spent walking at different cadences by males with COPD. In the current study, males with COPD spent less time walking at cadences $<100$ steps/min compared to healthy controls. High cadences ( $\geq 100$ steps $/ \mathrm{min}$ ) were considered the threshold for moderately intense physical activity in adults to optimize health benefits such as reductions in the risk of cardiovascular and metabolic disease. ${ }^{1,32}$ However, neither males with COPD nor the healthy controls in this study participated in walkingbased activity at cadences $\geq 100$ steps/min, which explains the lack of difference between these 2 groups at high cadences. These findings suggest that males with COPD as well as healthy controls in Saudi Arabia may be at risk of developing chronic diseases, such as cardiovascular and metabolic diseases. ${ }^{1}$ Therefore, in Saudi Arabia, awareness of the benefits of walking at a moderate intensity needs to be promoted among the general public as well as males with COPD. Studies that found increased physical activity in people with COPD frequently used strategies such as individual goal setting based on the collection of objective physical activity data, action and coping planning to overcome barriers, motivational interviews, and regular contact with healthcare professionals to increase motivation..$^{33,34}$ Therefore, these approaches need to be considered when implementing interventions that aim to increase the time Saudi nationals spend engaged in walkingbased physical activity and reduce the time they spend engaged in sedentary behavior.

The present study demonstrated moderate to strong associations between the components of D-12 and time spent engaged in walking-based activity and sedentary behavior. Notably, the associations between time engaged in walking-based activity and sedentary behavior and the emotional response component of the D-12 ( $\mathrm{r}: \geq-0.63$ and $\mathrm{r}: \geq 0.66$, respectively) were stronger than the associations with quality component of the D-12 ( $r: \geq-0.52$ and $r: \geq 0.46$, respectively). These findings indicate that males with COPD for whom emotional response to dyspnea is greater than the quality of the sensation spend fewer waking hours engaged in walking-based activity and more time engaged in sedentary behavior. These findings suggest that interventions such as cognitive behavioral therapy, which aim to minimize emotional response to dyspnea, may help increase the level of physical activity and reduce sedentary behavior in males with COPD. However, the coefficient of determination for the association between emotional response to dyspnea and time spent engaged in walking-based activity $\left(\mathrm{R}^{2}=0.40\right)$ or sedentary behavior $\left(\mathrm{R}^{2}=0.44\right)$ demonstrates that, at most, only $44 \%$ of the variance in either time spent engaged in walking-based activity or sedentary behavior is explained by emotional repose to dyspnea. Therefore, this finding suggests that other important factors, such as lower exercise capacity and higher rate of exacerbation in the past year, contribute to how much time males with COPD spend engaged in walking-based activity and sedentary behavior. ${ }^{4}$ 
This study had some limitations. It recruited a relatively small sample of males from a single location (Riyadh, Saudi Arabia), limiting the generalizability of the findings to other Arabic populations as well as to females. The cadence bands used in this study, which were proposed by the NHANES, could be a limitation because they have not been validated in a COPD population. ${ }^{27}$ Further, the present study measured only walking-based activity and omitted other forms of physical activity, such as cycling. However, walking is considered to be fundamental for human functioning and mobility. ${ }^{35}$ Defining the time spent engaged in sedentary behavior by grouping any one-minute epoch with a step count of zero could overestimate the amount of time engaged in sedentary behavior. Additionally, 6MWD data were not collected for healthy controls in this study. However, it is well known that healthy people exhibit higher exercise capacity than people with COPD. ${ }^{3,29}$ Also, comparison between groups in terms of functional exercise capacity was beyond the scope of this study.

In summary, males with COPD were less active and more sedentary than healthy controls. The quality and emotional response components of the D-12 were associated with walking-based activity and sedentary behavior in males with COPD. The findings of this study can help multidisciplinary initiatives identify strategies for optimizing the time spent engaged in walking-based activity and decreasing the time spend engaged in sedentary behavior in people with COPD.

Acknowledgment. The authors would like to thank Dr. Kevin Netto (Associate Professor, Director of Research, School of Physiotherapy and Exercise Science, Curtin University, Australia) for assistance with analysis of sedentary data.

\section{References}

1. Garber CE, Blissmer B, Deschenes MR, Franklin B, Lamonte MJ, Lee IM, et al. American College of Sports Medicine position stand. Quantity and quality of exercise for developing and maintaining cardiorespiratory, musculoskeletal, and neuromotor fitness in apparently healthy adults: guidance for prescribing exercise. Med Sci Sports Exerc 2011; 43: 1334-1359.

2. Troosters T, Sciurba F, Battaglia S, Langer D, Valluri SR, Martino L, et al. Physical inactivity in patients with COPD, a controlled multi-center pilot-study. Respir Med 2010; 104: 1005-1011.

3. Pitta F, Troosters T, Spruit MA, Probst VS, Decramer M, Gosselink R. Characteristics of physical activities in daily life in chronic obstructive pulmonary disease. Am J Respir Crit Care Med 2005; 171: 972-977.

4. Hartman JE, Boezen HM, de Greef MH, Ten Hacken NH. Physical and psychosocial factors associated with physical activity in patients with chronic obstructive pulmonary disease. Arch Phys Med Rehabil 2013; 94: 2396-2402.
5. Marshall SJ, Levy SS, Tudor-Locke CE, Kolkhorst FW, Wooten $\mathrm{KM}$, Ji M, et al. Translating physical activity Rrcommendations into a pedometer-based step goal: 3000 steps in 30 minutes. Am J Prev Med 2009; 36: 410-415.

6. Tudor-Locke C, Sisson SB, Collova T, Lee SM, Swan PD. Pedometer-determined step count guidelines for classifying walking intensity in a young ostensibly healthy population. Can J Appl Physiol 2005; 30: 666-676.

7. Rowe D, Welk G, Heil D, Mahar M, Kemble C, Calabro $\mathrm{M}$, et al. Stride rate recommendations for moderate-intensity walking. Med Sci Sports Exerc 2011; 43: 312-318.

8. Abel M, Hannon J, Mullineaux D, Beighle A. Determination of step rate thresholds corresponding to physical activity intensity classifications in adults. J Phys Act Health 2011; 8: 45-51.

9. Biswas A, Oh PI, Faulkner GE, Bajaj RR, Silver MA, Mitchell MS, et al. Sedentary time and its association with risk for disease incidence, mortality, and hospitalization in adults: a systematic review and meta-analysis. Ann Intern Med 2015; 162: 123-132.

10. Furlanetto KC, Donária L, Schneider LP, Lopes JR, Ribeiro $\mathrm{M}$, Fernandes KB, et al. Sedentary behavior is an independent predictor of mortality in subjects with COPD. Respir Care 2017; 62: 579-587.

11. Vorrink SN, Kort HS, Troosters T, Lammers J. Level of daily physical activity in individuals with COPD compared with healthy controls. Respir Res 2011; 12: 4-8.

12. Al-Hazzaa HM. Health-enhancing physical activity among Saudi adults using the international physical activity questionnaire (IPAQ). Public Health Nutr 2007; 10: 59-64.

13. Mandil AM, Alfurayh NA, Aljebreen MA, Aldukhi SA. Physical activity and major non-communicable diseases among physicians in Central Saudi Arabia. Saudi Med J 2016; 37: 1243-1250.

14. Bajamal E, Robbins LB, Ling J, Smith B, Pfeiffer KA, Sharma D. Physical activity among female adolescents in Jeddah, Saudi Arabia: a health promotion model-based path analysis. Nurs Res 2017; 66: 473-482.

15. Al-Nozha MM, Al-Hazzaa HM, Arafah MR, Al-Khadra A, Al-Mazrou YY, Al-Maatouq MA, et al. Prevalence of physical activity and inactivity among Saudis aged 30-70 years: a population-based cross-sectional study. Saudi Med J 2007; 28 : 559-568.

16. Bonnefoy M, Normand S, Pachiaudi C, Lacour JR, Laville M, Kostka T. Simultaneous validation of ten physical activity questionnaires in older men: a doubly labeled water study. $\mathrm{J} \mathrm{Am}$ Geriatr Soc 2001; 49: 28-35.

17. Vogelmeier CF, Criner GJ, Martinez FJ, Anzueto A, Barnes PJ, Bourbeau J, et al. Global strategy for the diagnosis, management and prevention of chronic obstructive lung disease 2017 report. Respirology 2017; 22: 575-601.

18. Cindy Ng LW, Jenkins S, Hill K. Accuracy and responsiveness of the stepwatch activity monitor and ActivPAL in patients with COPD when walking with and without a rollator. Disabil Rehabil 2012; 34: 1317-1322.

19. Alyami MM, Jenkins SC, Lababidi H, Hill K. Reliability and validity of an arabic version of the dyspnea-12 questionnaire for Saudi nationals with chronic obstructive pulmonary disease. Ann Thorac Med 2015; 10: 112-117.

20. Miller MR, Hankinson J, Brusasco V, Burgos F, Casaburi R, Coates A, et al. Standardisation of spirometry. Eur Respir J 2005; 26: 319-338.

21. Al Ghobain MO, Alhamad EH, Alorainy HS, Al Hazmi M, Al Moamary MS, Al-Hajjaj MS, et al. Spirometric reference values for healthy nonsmoking Saudi adults. Clin Respir J 2014; 8: 72-78. 
22. Holland AE, Spruit MA, Troosters T, Puhan MA, Pepin V, Saey $D$, et al. An official European Respiratory Society/American Thoracic Society technical standard: field walking tests in chronic respiratory disease. Eur Respir J 2014; 44: 1428-1446.

23. Casanova C, Celli B, Barria P, Casas A, Cote C, De Torres J, et al. The 6-min walk distance in healthy subjects: reference standards from seven countries. Eur Respir J 201 1; 37: 150-156.

24. Lores V, García-Río F, Rojo B, Alcolea S, Mediano O. Recording the daily physical activity of COPD patients with an accelerometer: an analysis of agreement and repeatability. Arch Bronconeumol 2006; 42: 627-632.

25. Watz H, Waschki B, Meyer T, Magnussen H. Physical activity in patients with COPD. Eur Respir J 2009; 33: 262-272.

26. Hart TL, Swartz AM, Cashin SE, Strath SJ. How many days of monitoring predict physical activity and sedentary behaviour in older adults? Int J Behav Nutr Phys Act 2011; 8: 62.

27. Tudor-Locke C, Camhi SM, Leonardi C, Johnson WD, Katzmarzyk PT, Earnest CP, et al. Patterns of adult stepping cadence in the 2005-2006 NHANES. Prev Med 2011; 53: 178-181.

28. Downs J, Leonard H, Wong K, Newton N, Hill K. Quantification of walking-based physical activity and sedentary time in individuals with Rett syndrome. Dev Med Child Neurol 2017; 59: 605-611.
29. Hernandes NA, Teixeira C, Probst VS, Brunetto AF, Ramos EMC, Pitta F. Profile of the level of physical activity in the daily lives of patients with COPD in Brazil. J Bras Pneumol 2009; 35: 949-956.

30. Hill K, Dolmage TE, Woon L, Coutts D, Goldstein R, Brooks D. Defining the relationship between average daily energy expenditure and field-based walking tests and aerobic reserve in COPD. Chest 2012; 141: 406-412.

31. Zwerink M, van der Palen J, van der Valk P, Brusse-Keizer M, Effing T. Relationship between daily physical activity and exercise capacity in patients with COPD. Respir Med 2013; 107: 242-248.

32. Tudor-Locke C, Rowe DA. Using cadence to study free-living ambulatory behaviour. Sports Med 2012; 42: 381-398.

33. Kawagoshi A, Kiyokawa N, Sugawara K, Takahashi H, Sakata S, Satake M, et al. Effects of low-intensity exercise and home-based pulmonary rehabilitation with pedometer feedback on physical activity in elderly patients with chronic obstructive pulmonary disease. Respir Med 2015; 109: 364-371.

34. Mendoza L, Horta P, Espinoza J, Aguilera M, Balmaceda N, Castro A, et al. Pedometers to enhance physical activity in COPD: a randomised controlled trial. Eur Respir J 2015; 45 : 347-354.

35. Ham SA, Kruger J, Tudor-Locke C. Participation by US adults in sports, exercise, and recreational physical activities. J Phys Act Health 2009; 6: 6-14. 